\title{
Photoinduced femtosecond formation of ferromagnetism in a strongly correlated antiferromagentic manganite
}

\author{
T. Li, ${ }^{1,2}$ A. Patz, ${ }^{1,2}$ J. Yan, ${ }^{1}$ T. A. Lograsso, ${ }^{1}$ I. E. Perakis ${ }^{3}$ and J. Wang, ${ }^{1,2}$ \\ ${ }^{1}$ Department of Physics and Astronomy, Iowa State University, USA \\ ${ }^{2}$ Ames Laboratory - USDOE, USA \\ ${ }^{3}$ Department of Physics, University of Crete, Greece
}

\begin{abstract}
We report a pump threshold behavior in fs photoinduced magnetization in a strongly correlated manganite, which indicates the establishment of thermally-inaccessible ferromagnetic ground state and buildup of new magnetic order parameters at fs time scales
\end{abstract}

\section{INTRODUCTION}

There has been strong current interest to manipulate collective spin ordering and induce magnetic phase transitions in their highly non-equilibrium, non-thermal states at femtosecond time scales. Such processes offer opportunities to exceed the upper limit of the magnetic switching speed (0.1-10 $\mathrm{GHz}$ ) in modern magneto-optical recording industry and magnetic storage/logic devices. In strong contrast with the conventional, thermally-induced phase transitions, transient structures of nonequilibrium phases and spin states at femtosecond time scales are formed by collective interactions and/or coherent couplings, which are much less studied and poorly understood. Recently, there is growing evidence that non-thermal control of magnetism at fs timescales is feasible, which may involve direct coupling between the light field and/or photoexcited non-thermal carriers and collective spins, even faster than one oscillation cycle of phonons. Thus far, searching for such femtosecond magnetism has been explored in conventional, more "weakly-correlated" spin systems such as metals [1], band insulators [2] and semiconductors [3-5].

Strongly correlated electrons represent a unique class of materials to explore femtosecond magnetism. Strong interactions among particles in these complex magnetic systems can favor competing types of magnetic ground states and orders. In these materials, there is growing importance for not only thermally accessible magnetic states but also hidden magnetic states that cannot be observed through conventional temperature-tuning, offering potential strategies for searching these states via non-thermal fs photoinduced phase transitions. However, experimental evidence for photoinduced fs magnetic phase transition in these materials has been either absent or at longer pico- or nanosecond time scales, governed mainly by spin-phonon interactions [6]. One key experimental observation that is still missing is a threshold behavior at fs time scales associated with non-thermal, magnetic phase transitions. Moreover, almost all prior theoretical understandings of this highly non-equilibrium regime have been based on phenomenological thermodynamic models.

Here using ultrafast two-color magnetic circular dichroism (MCD) spectroscopy, we have, for the first time, observed a substantial photoinduced magnetization enhancement within at fs time scales above a threshold pump fluence and at low temperature in a strongly correlated

This is an Open Access article distributed under the terms of the Creative Commons Attribution License 2.0, which permits unrestricted use, distribution, and reproduction in any medium, provided the original work is properly cited. 
antiferromagnetic manganite $\operatorname{Pr}_{0.7} \mathrm{Ca}_{0.3} \mathrm{MnO}_{3}$. Such a photoinduced critical behavior vanishes at elevated temperature. Our results show a photoinduced, non-thermal ultrafast antiferromagnetic to ferromagnetic phase transition, demonstrating particularly, that one can reveal a hidden, thermally inaccessible ground state at fs time scales.

\section{EXPERIMENTAL METHODS}

We perform UV pump and NIR probe MCD spectroscopy on a model Mott insulator $\mathrm{Pr}_{0.7} \mathrm{Ca}_{0.3} \mathrm{MnO}_{3}$ (PCMO) [Figure 1(a)]. It exhibits a layered structure which is coupled by superexchange interaction that leads to a quasi-2D antiferromagnetic ordering. The "apparent" ground state is always insulating as a function of temperature, which exhibits charge/orbital ordering $(\mathrm{CO} / \mathrm{OO})$ and antiferromagnetic phases at low temperature, shown in magneto-transport diagram in Figure 1(b). It has been established that applying a magnetic field or pressure, but not temperature, will lead to the "melting" of a charge-orbital-ordered insulator to a ferromagnetic metal, a thermallyinaccessible hidden ground state.

(a)

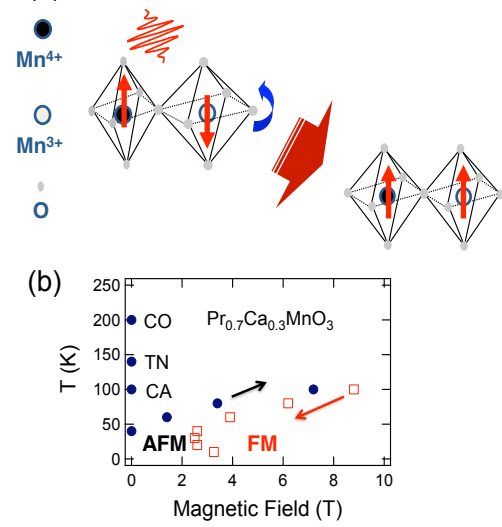

(c)

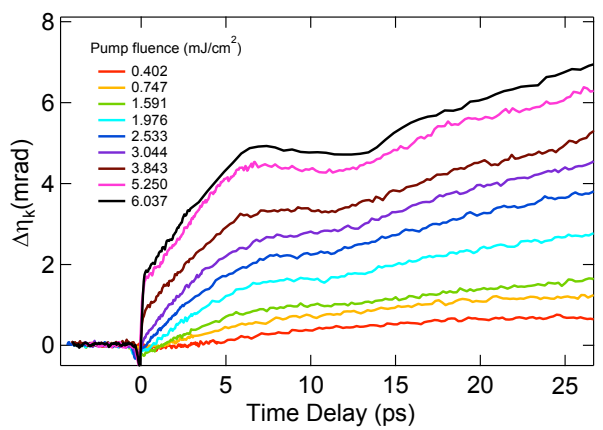

Fig. 1.(a) The experimental schematics for exploring an ultrafast photoinduced antiferro- (AFM) to ferromagnetic (FM) phase transition in $\operatorname{Pr}{ }_{0.7} \mathrm{Ca}_{0.3} \mathrm{MnO}_{3}$. (b) Temperature vs. magnetic-field phase diagram shows a magnetic field induced AFM to FM transition. TN: Neel temperature; CO:chargeorder; CA: canted AFM; (c) Photoinduced transitent magnetization changes obtained by two-color, NIR pump/UV probe MCD spectroscopy. A threshold behavior is seen $\sim 3 \mathrm{~mJ} / \mathrm{cm}^{2}$ for significant magnetization enhancement within the first $180 \mathrm{fs}$, suggesting a photoinduced magnetic phase transition.

\section{RESULTS AND DISCUSSIONS}

Typical data showing the general temporal profile of the photoinduced ellipticity change $\Delta \eta_{K}$, are shown in Figure 1(c), for the first $25 \mathrm{ps}$. The sign of $\Delta \eta_{K}$ is always positive, indicating transient photoinduced magnetization enhancement. Distinct temporal regimes can be identified with different pump fluence dependence: at low pump fluence, there is a slow, ps gradual enhancement (up to $\sim 30 \mathrm{ps}$ ). In strong contrast to this, at high pump fluence, an initial fast, fs increase in magnetization is followed by a slow ps enhancement with periodical oscillations. Most intriguingly, the pump fluence dependence shows a distinctive threshold, "step-function-like" enhancement in the magnetization, which is reminiscent of critical behaviors for ultrafast photoinduced antiferro- to ferromagnetic phase transitions. These results clearly demonstrate the collapse of the correlation gap in the antiferromagnetic states and build up of a new ferromagnetic order parameter at $180 \mathrm{fs}$ time scales. 
We attribute the observed ultrafast switching between antiferro- to ferromagnetic state to the inter-chain charge transfer excitation, as discussed below. Before the ultrafast photo excitation, the spins are characterized by an ensemble of $1 \mathrm{D}$ spin chains with a ferromagnetic alignment along the orbital zigzag chains and an antiferromagnetic coupling between the adjacent chains. At pump-probe delays $\Delta \mathrm{t} \sim 0 \mathrm{fs}$, the femtosecond laser pulses generates inter- and intra-chain charge-transfer excitations between $\mathrm{Mn}^{3+}$ and $\mathrm{Mn}^{4+}$ sites. Particularly, the quantum spin nature allows the local interchain hopping with opposite spin alignment, which is weak in the ground state and forbidden for classical spins because of the large Hund's energy and small orbital overlap between two chains. Below the threshold of the magnetic phase transition, the inter-spin-chain charge transfer results in an itinerant electron antiferromagnetically coupled with a ferromagnetic spin chain, which leads to the formation of magnetic polarons - a collective state favouring short range ferromagnetic alignment. The ps, slow magnetization rise observed reflects dynamics of such polaron-like collective photoexcitations, which show comparable formation time to those in magnetic semiconductors [7-8]. However, these magnetic polaron-like states are uncorrelated below the excitation threshold where a long-range ferromagnetic phase is absent. Increasing the photoexcitation from the linear to the highly nonlinear regime of the $\mathrm{CO} / \mathrm{OO}$ melting, the sizes of the ferromagnetic clusters grow nonlinearly and exceed the critical size for percolative phase transition at the threshold pump fluence. A global conducting path of $\mathrm{e}_{\mathrm{g}}$ electrons emerges and leads to a long range correlation among spin clusters, which reveals a hidden, thermally inaccessible ferromagnetic phase at fs time scale.

\section{CONCLUSIONS}

In summary, we report a pump threshold behavior in fs photoinduced magnetization in a strongly correlated manganite, which indicates the establishment of thermally-inaccessible ferromagnetic ground state and build-up of new magnetic order parameters at fs time scales.

Acknowledgements. This work was also supported by the National Science Foundation Contract No. DMR-1055352. Material synthesis at the Ames Laboratory was supported by the U.S. Department of Energy-Basic Energy Sciences under Contract No. DE-AC02-7CH11358.

\section{References}

1. A. V. Kimel, A. Kirilyuk, P. A. Usachev, R. V. Pisarev, A. M. Balbashov and Th. Rasing, Nature 435, 655-657 (2005).

2. Jean-Yves Bigot, Mircea Vomir and Eric Beaurepaire, Nature Physics 5, 515 - 520 (2009)

3. J. Wang, I. Cotoros, D. S. Chemla, X. Liu, J. K. Furdyna, J. Chovan, and I. E. Perakis, Appl. Phys. Lett. 94, 021101 (2009)

4. M. D. Kapetanakis, J. Wang, and I. E. Perakis, "Femtosecond All-Optical Modulation of Collective Spin in the (Ga,Mn)As Ferromagnet," J. Opt. Soc. Am. B 29, A95-A102 (2011)

5. M. D. Kapetanakis, I. E. Perakis, K. J. Wickey, C. Piermarocchi, and J. Wang, "Femtosecond Coherent Control of Spins in (Ga,Mn)As Ferromagnetic Semiconductors Using Light," Physical Review Letters, 103, 047404 (2009)

6. S. A. McGill, R. I. Miller, O. N. Torrens, A. Mamchik, I-Wei Chen, and J. M. Kikkawa, Phys. Rev. B 71, 075117 (2005).

7. D. D. Awschalom et al., Phys. Rev. Lett. 55, 1128 (1985).

8. J. Wang, I. Cotoros, K. M. Dani, X. Liu, J. K. Furdyna, and D. S. Chemla, Physical Review Letters, 98, 217401 (2007) 